\title{
Universidad de Guadalajara
}

\author{
Ramón Willman Zamora*
}

RESUMEN: Se presenta información sobre la investigación en temas de nanociencia y nanotecnología en la Universidad de Guadalajara incluyendo recursos humanos, infraestructura, líneas de investigación, proyectos con financiamiento externo, instrumentos en propiedad intelectual solicitados y oferta de formación en nanociencias.

PALABRAS CLAVE: Investigación, nanociencia, nanotecnología, Universidad de Guadalajara, patentes, posgrado.

ABSTRACT: Information about the subjects of research on nanoscience and nanotechnology in the University of Guadalajara is presented. The human resource training, infrastructure, lines of research, projects undertaken in the area, patents requested and teaching activities, are reported.

KEYWORDS: Research, nanoscience, nanotechnology, University of Guadalajara, patents, postgraduate studies.

En este apartado presentaremos un breve pero significativo artículo de divulgación de la nanociencia y nanotecnología en la Universidad de Guadalajara tomando en cuenta los siguientes ejes: recursos humanos, infraestructura, líneas de investigación, proyectos con financiamiento externo, instrumentos en propiedad intelectual solicitados y oferta de formación en nanociencias.

\section{Recursos humanos}

La Universidad de Guadalajara cuenta con 25 profesores que trabajan líneas de investigación afines a la nanociencia y nanotecnología, de éstos, 21 pertenecen al Sistema Nacional de Investigadores con los siguientes niveles de reconocimiento, 3 son candidatos, 16 pertenecen al nivel uno y 1 cuenta con el reconocimiento del nivel dos. De los 25 profesores, 23 de ellos se encuentran agrupados en 5 cuerpos académicos (tabla 1 ).

\section{Líneas de investigación, desarrollo e innovación relacionadas con la $\mathrm{NyN}$}

Las líneas de investigación que se trabajan en cuanto a nanocienca y nanotecnología son las siguientes:

Recibido: 24 de junio 2016. Aceptado: 25 de octubre de 2016.

* Universidad de Guadalajara, Coordinación General Académica, Coordinación de Investigación y Posgrado. Correspondencia: (Ramon.Willman@redudg.udg.mx). 
TABLA 1. Profesores investigadores pertenecientes a cuerpos académicos que desarrollan líneas de investigación en nanotecnología.

\begin{tabular}{|c|c|c|c|c|}
\hline $\begin{array}{l}\text { CENTRO } \\
\text { UNIVERSITARIO }\end{array}$ & $\begin{array}{l}\text { NÚMERO } \\
\text { CUERPO } \\
\text { ACADÉMICO }\end{array}$ & $\begin{array}{l}\text { NOMBRE } \\
\text { DEL CUERPO } \\
\text { ACADÉMICO }\end{array}$ & $\begin{array}{c}\text { NÚMERO } \\
\text { INTEGRANTES }\end{array}$ & $\begin{array}{c}\text { NÚMERO } \\
\text { SNI }\end{array}$ \\
\hline CUCEI & UDG-CA-158 & $\begin{array}{l}\text { Ciencia y tecnología de } \\
\text { materiales } \\
\text { lignocelulósicos }\end{array}$ & 4 & 3 \\
\hline CUVALLES & UDG-CA-583 & $\begin{array}{l}\text { Ciencia de } \\
\text { nanomateriales y } \\
\text { materia condensada }\end{array}$ & 4 & 4 \\
\hline CULAGOS & UDG-CA-674 & $\begin{array}{l}\text { Tecnologías de } \\
\text { materiales }\end{array}$ & 4 & 4 \\
\hline CUCIENEGA & UDG-CA-685 & $\begin{array}{l}\text { Nanomateriales } \\
\text { poliméricos y catalíticos }\end{array}$ & 5 & 3 \\
\hline \multirow[t]{2}{*}{ CUVALLES } & UDG-CA-792 & $\begin{array}{l}\text { Propiedades físicas y } \\
\text { químicas de sistemas } \\
\text { nanoestructurados }\end{array}$ & 6 & 5 \\
\hline & & Total & 23 & 19 \\
\hline
\end{tabular}

Fuente: Elaboración propia.

- Fabricación y caracterización de materiales nanoestructurados en aplicaciones tecnológicas.

- Síntesis y caracterización fiscoquímica de materiales micro y nanoestrcutruados.

- Preparación y caracterización de materiales y nanomateriales compositos poliméricos.

- Cálculos de las propiedades de nanotubos de carbono en contacto con diferentes solventes y nanopartículas.

- Nanofísica y nanoquímica.

- Catálisis y nanotecnología de los materiales catalíticos.

- Citotoxicidad: productos bioactivos y nanomateriales (t6).

Algunos de los proyectos que se desarrollan actualmente en la Universidad en el campo de las NyN son los siguientes:

- Reología de fluidos complejos: micelas gigantes, polímeros asociativos y nanocompuestos: el objetivo del proyecto es estudiar sistemáticamente los estados transitorios reológicos de micelas gigantes, polímeros, nanocompuestos y suspensiones electroactivas aplicando campos eléctricos constantes y oscilatorios.

- Síntesis y caracterización de física de materiales ferromagnéticos, ferroeléctricos y ferromagnetoeléctricos: bajo una perspec- 
tiva nanotecnológica: este proyecto busca realizar la síntesis y caracterización física de nuevos materiales con propiedades ferromagnéticas, ferroeléctricas y ferromagnetoeléctricas a escala nanométrica.

- Generación de películas y nanopartículas antimicrobiales a partir de compuestos de origen vegetal, mediante el uso de plasmas fríos: el objetivo del proyecto es realizar la síntesis asistida por plasma de películas delgadas, y nanopartículas con características antimicrobiales, a partir de compuestos de origen vegetal, y caracterización de las mismas.

- Microemulsión: establecimiento de una nueva ruta de síntesis de nanopartículas de compuestos coordinados mediante el uso de plantillas formadas por sistemas micelares.

- Obtención de materiales nanoestructurados con propiedades fotocatalíticas: desarrollar diversos materiales nanoestructurados (nanotubos, nanoalambres) con propiedades fotocatalíticas para su aplicación en desinfección biológica y remoción de orgánicos contaminantes de agua.

- Remoción de cromo de corrientes acuosas industriales utilizando materiales nanoestructurados funcionalizados: mediante el proceso sol-gel para la síntesis de adsorbentes para la extracción de $\mathrm{Cr}$ (III, VI) de corrientes acuosas industriales. Caracterización de adsorbentes por medio de experimentos de adsorción de $\mathrm{Cr}$ (III, VI) y diversas técnicas espectroscópicas, obteniendo un mejor entendimiento de los procesos de adsorción por medio de modelos matemáticos.

- Desarrollo de nanosistemas poliméricos bioactivos para aplicaciones biomédicas: el objetivo es lograr la síntesis de nanopartículas poliméricas inteligentes, capaces de responder ante estímulos externos, específicamente ante variaciones en los valores de $\mathrm{PH}$ y variaciones en la temperatura.

- Estudio de materiales semiconductores nanoestructurados para su uso como sensores ambientales de CO: se busca la preparación de materiales inorgánicos nanoestructurados, a través de diversas técnicas, así como su análisis mediante difracción de rayos $\mathrm{X}$, microscopía electrónica, FTIR, etc. Medición de propiedades eléctricas y modelado de las mismas.

\section{Infraestructura}

La Universidad de Guadalajara se compone de 15 Centros Universitarios, 6 de los cuales se localizan en la Zona Metropolitana de Guadalajara (ZMG) y 9 ubicados en el interior del estado; los recursos humanos yla infraestructura para el desarrollo de la nanotecnología se encuentran ahí distribuidos. A 
Mundo Nano | Catálogo Nacional | vol. 9, No. 17, julio-diciembre, 2016 | www.mundonano.unam.mx

TABLA 2. Laboratorios de la Universidad de Guadalajara destinados a NyN.

\begin{tabular}{|c|c|c|}
\hline CENTRO UNIVERSITARIO & NOMBRE DEL LABORATORIO & MUNICIPIO \\
\hline $\begin{array}{l}\text { Centro Universitario de } \\
\text { Ciencias Exactas e Ingenierías } \\
\text { (CUCEI) }\end{array}$ & $\begin{array}{l}\text { Laboratorio de Biomateriales y } \\
\text { Nanotecnología }\end{array}$ & Guadalajara \\
\hline \multirow{2}{*}{$\begin{array}{l}\text { Centro Universitario de Tonalá } \\
\text { (CUTONALA) }\end{array}$} & Laboratorio de Nanotecnologías & Tonalá \\
\hline & $\begin{array}{l}\text { Laboratorio de Microscopía e } \\
\text { Instrumentación }\end{array}$ & Tonalá \\
\hline \multirow[t]{2}{*}{$\begin{array}{l}\text { Centro Universitario de los } \\
\text { Valles (CUVALLES) }\end{array}$} & $\begin{array}{l}\text { Centro de Investigaciones en } \\
\text { Nanociencias }\end{array}$ & Ameca \\
\hline & $\begin{array}{l}\text { Laboratorio de Desarrollo y } \\
\text { Caracterización de Materiales }\end{array}$ & Ameca \\
\hline $\begin{array}{l}\text { Centro Universitario de la } \\
\text { Ciénega (CUCIENEGA) }\end{array}$ & $\begin{array}{l}\text { Laboratorio de Nanomateriales } \\
\text { Catalíticos }\end{array}$ & Ocotlán \\
\hline $\begin{array}{l}\text { Centro Universitario de los } \\
\text { Altos (CUALTOS) }\end{array}$ & $\begin{array}{l}\text { Laboratorio de Biotecnología y } \\
\text { Catálisis }\end{array}$ & Tepatitlán \\
\hline
\end{tabular}

Fuente: Elaboración propia.

junio de 2016, la Universidad cuenta con 7 laboratorios que realizan investigación básica y aplicada en nanociencia, tres de ellos localizados en municipios de la Zona Metropolitana (tabla 2).

\section{Instrumentos de protección de propiedad intelectual solicitados}

En la actualidad se tienen dos solicitudes ingresadas al Instituto Mexicano de la Propiedad Industrial (IMPI) las cuales se encuentran próximas a publicarse en la Gaceta del IMPI; la primera solicitud ${ }^{1}$ titulada "Método de separación, extracción y liberación de compuestos no polares de origen vegetal por medio de nanopartículas de hidróxidos dobles laminares que contienen dichos compuestos" relacionada con la separación de compuestos no polares de origen vegetal mediante la síntesis de hidróxidos dobles laminares in situ en un material vegetal que contiene dicho compuesto no polar y un método para liberar de forma selectiva el compuesto separado, las ventajas que presenta esa invención son:

- Minimiza los inconvenientes que presentan los métodos de separación, liberación y producción de compuestos de origen vegetal utilizados en la actualidad e incrementa la estabilidad de estos

1 Esta solicitud fue publicada en la Gaceta de IMPI del mes de julio de 2016, corresponde al número $\mathrm{Mx} / \mathrm{a} / 2015 / 017358$. 
compuestos sensibles a factores ambientales como temperatura, presión, humedad, luz solar, ataque microbiano, oxidación, entre otros.

- Provee compuestos no polares de origen vegetal con una mayor estabilidad y resistencia a la fotodegradación para aumentar la vida de anaquel y almacenamiento de los mismos.

- Provee métodos de separación/extracción más simples, significativamente económicos y no involucra solventes ni condiciones agresivas que puedan comprometer las propiedades de dicho compuesto no polar de origen vegetal y se pueda obtener con un alto grado de pureza.

Los autores de esta invención son Gregorio Guadalupe Carbajal Arízaga y Daniel Escobar Hernández junto con el alumno Michel Jorgelina Montoya Gutiérrez.

La segunda solicitud" titulada "Partículas de hidróxidos dobles laminares neutralizadas con aniones polares con enlace PI como huéspedes para intercalación de moléculas no iónicas y el método de intercalación" la cual provee partículas de HDL neutralizadas con iones folato con moléculas neutras intercaladas entre sus láminas mediante procedimientos de coprecipitación, para la preparación de nuevas formas farmacéuticas para su uso como un medicamento útil para el tratamiento de cáncer, dentro de las ventajas que presenta esa invención se encuentran:

- Permitir la transportación efectiva de fármacos cuyas moléculas son neutras y especialmente aquellos medicamentos útiles para el tratamiento del cáncer (como el imidazol y la ciclofosfamida).

- Proteger el fármaco al dar estabilidad térmica y mecánica a las moléculas.

- Liberar efectivamente las moléculas y mejorar la disolución del fármaco.

Esta invención fue desarrollada por los profesores Gregorio Guadalupe Carbajal Arízaga, Ana María Puebla Pérez y los alumnos Cecilia Sánchez Jiménez y Luis Manuel Flores Alonso.

\section{Oferta educativa en NyN}

En materia de formación en nanociencias y nanotecnología, la Universidad de Guadalajara a través del Centro Universitario de Tonalá oferta la licenciatura e ingeniería en nanotecnología, cuyos objetivos de formación se enlistan a continuación:

2 Esta solicitud fue publicada en la Gaceta de IMPI del mes de julio de 2016, corresponde al número $\mathrm{Mx} / \mathrm{a} / 2015 / 017355$. 
- Proporcionar al estudiante una cultura científica, tecnológica y humanística.

- Una formación metodológica que lo prepare para adaptar e incorporar los avances científicos y tecnológicos a su campo profesional.

- Fomentar una sólida formación interdisciplinaria en nanociencias y nanotecnología.

- Que el egresado sea capaz de generar nuevas propiedades a base de la manipulación atómica y molecular de la materia para aplicaciones en sectores estratégicos y, en particular, para resolver problemas prioritarios actuales a nivel mundial en agua, energía, salud y medio ambiente.

En materia de posgrado tenemos maestrías y doctorado con orientaciones en nanotecnología como lo son:

- La maestría y doctorado en ciencias en ingeniería química; ambos programas cuentan con reconocimiento del Programa Nacional de Posgrados de Calidad (PNPC); se imparten en el Centro Universitario de Ciencias Exactas e Ingenierías.

- La maestría y doctorado en ciencia y tecnología; ambos con reconocimiento del PNPC; se imparten en el Centro Universitario de los Lagos.

- La maestría y doctorado en ciencias físico-matemáticas; ambos con reconocimiento del PNPC; se imparten en el Centro Universitario de los Valles.

- La maestría y doctorado en ciencias; se imparten en el Centro Universitario de la Ciénega. 